\title{
Cryptanalysis of an Untraceable Temporal-Credential-Based Two-Factor Authentication Scheme Using ECC for Wireless Sensor Networks
}

\author{
Shu-Ying Yang \\ Department of basic, Shandong Women's University, \\ Jinan, China \\ E-mail: ysystudy2005@163.com
}

\author{
Cheng-Bo Xu \\ School of Mathematical Sciences, University of Jinan, \\ Jinan, China \\ E-mail: cbqysy@163.com
}

\begin{abstract}
Authentication and key agreement scheme is an important mechanism for legal users to access the services of wireless sensor network. However, the design of authentication and key agreement schemes in WSNs is still quite a challenging problem. In this paper, we analyze an untraceable temporalcredential-based two-factor authentication scheme using ECC for WSNs proposed by Jiang et al. in 2016, and point out the scheme can not resist malicious user impersonation attack, sensor node capture attack and suffer from forward security problem, low efficiency problem, no password change phase problem and time synchronization problem.
\end{abstract}

Keywords-authentication; password; smart card; session key agreement; wireless sensor netwoks

\section{INTRODUCTION}

Nowadays, wireless sensor networks (WSNs) are the first choices for a wide range of real-time monitoring applications, such as health care, environmental monitoring, traffic monitoring, etc. In WSNs, data collected by sensor nodes sometimes contain valuable and confidential information that only authorized users are allowed to access. As yet, the design of user authentication and key agreement scheme for resource deficient wirless sensor networks has been substantially addressed by various researchers.

In 2009, Das [1] first proposed a two-factor authentication scheme for wieless sensor networks using smart card, which leads user authentication for WSNs to a new direction. In this direction, a dozen of authentication schemes have been presented to eliminate the security weaknesses of the earlier ones [2-7]. Das claimed his scheme to be free from the security problems such as stolenverifier, many logged-in-users with the same identity, guessing, impersonation and replay attacks. In 2010, He et al. [2] pointed out that Das's scheme does not resist impersonation attack, privileged insider attack and lack of password update mechanism. During the same time, Khan and Alghathbar [3] showed that Das's scheme susceptible to gateway node bypassing attack and privileged insider attack and proposed an improved scheme. In 2013, Xue et al. [8] proposed an authentication scheme based on the concept of temporal credential for wireless sensor networks. They claimed that their scheme provides identity and password protection, and is immune to stolen smart card attack. Unfortunately, this scheme was pointed out that it is susceptible to many kinds of attacks such as offline password guessing attack, sensor node impersonation attack et al. by $\mathrm{He}$ et al. [9]. Based on these, He et al. improved Xue et al.'s scheme. However, Jiang et al. [10] revealed that He et al.'s scheme is susceptible to stolen smart card attack and prone to tracking attack, and proposed an improved scheme in 2016.

In this paper, we will show that Jiang et al.'s scheme [10] is also not secure and vulnerable to malicious user impersonation attack and node capture attack and suffer from forward security problem, low efficiency problem, no password change phase problem and time synchronization problem.

The rest of this paper is organized as follows: in section 2 , we briefly review Jiang et al.'s scheme. Section 3 points out the weaknesses of Jiang et al.'s scheme. Finally, we draw our conclusion in section 4 .

The notations used throughout this paper are summarized in Table 1.

\section{TABLE I. NOTATIONS}

\begin{tabular}{ll}
\hline$I D_{i}, P W_{i}$ & The identity and password of user $U_{i}$ \\
$S I D_{j}$ & Sensor node identity \\
$D I D_{i}, D I D_{G W N}$ & A dynamic identity of $U_{i}$ and $G W N$ \\
$K_{G W N-U}, K_{G W N-S}$ & Master keys only known to $G W N$ \\
$S K_{i j}$ & The session key agreed between $U_{i}$ and \\
& $S_{j}$ \\
$P T C_{i}$ & The protected temporal credential of \\
& $U_{i}$ \\
$T C_{i}, T C_{j}$ & The temporal credential of $U_{i}$ and $S_{j}$ \\
$T E_{i}$ & The expiration time of a user's \\
$T S$ & temporal credential \\
$K_{i}, K_{j}$ & The current timestamp \\
$h(\cdot)$ & Keys generated by $U_{i}$ and $S_{j}$ \\
$\oplus$ & A secure one-way hash function \\
$\|$ & The bitwise exclusive-or operation \\
\hline & Message concatenation operation \\
\hline
\end{tabular}

\section{REVIEW OF JIANG ET AL.'S SCHEME}

In this section, we briefly review the Jiang et al.'s scheme [10]. Their scheme includes three phases: registration phase, login phase and authentication phase; and involves three entities: users, gate-way node $(\mathrm{GW})$ and sensor nodes. 


\section{A. Registration Phase}

In this phase, $G W N$ selects the additive group $G$ generated by a point $P$ with a large prime order $n$ on an ellipic curve $E$. Then $G W N$ randomly generates a number $x$ as its private key and compoutes $y=x P$ as the public key. Finally, $G W N$ stores $x$ and publishes the system parameters $\{E, G, P, y\}$.

1) Registration phase for users

Assumpting that user $U_{i}$ shares a password $P W_{i}$ with $G W N$, which maintains the values $\left\{I D_{i}, H\left(P W_{i}\right)\right\}$. The details of this phase are reviewed as follows.

- Step 1: $U_{i}$ inputs the old password $P W_{i}$, and chooses a new one $P W_{i}^{\text {new }}$. Then he generates two random numbers $a, r_{i} \in Z_{p-1}^{*}$, and computes $I V_{i}=H\left(T S_{1}\left\|H\left(P W_{i}\right)\right\| A\left\|A^{\prime}\right\|\right.$

$$
\begin{aligned}
& \left.H\left(P W_{i}^{\text {new }}\left\|I D_{i}\right\| r_{i}\right)\right) \quad \text { an } T P W_{i}=H\left(P W_{i}^{\text {new }}\left\|I D_{i}\right\| r_{i}\right) \oplus \\
& H\left(T S_{1}\left\|H\left(P W_{i}\right)\right\| A \| A^{\prime}\right) \quad, \quad \text { where } \quad A=a P, \\
& A^{\prime}=a y=a x P \quad U_{i} \text { submits }\left\{I D_{i}, T S_{1}, V I_{i}, T P W_{i}, A\right\} \\
& \text { to } G W N .
\end{aligned}
$$

- Step 2: Upon receiving the message, $G W N$ verifies the validity of $T S_{1}$. Then, $G W N$ retrieves $H\left(P W_{i}\right)$ according to $I D_{i} \quad, \quad$ computes $H\left(P W_{i}^{\text {new }}\left\|I D_{i}\right\| r_{i}\right)=T P W_{i} \oplus H\left(T S_{1} \|\right.$

$\left.H\left(P W_{i}\right)\|A\| A^{\prime \prime}\right)$, and checks whether $V I_{i}$ and $H\left(T S_{1}\left\|H\left(P W_{i}\right)\right\| A\left\|A^{\prime}\right\| H\left(P W_{i}^{\text {new }}\left\|I D_{i}\right\| r_{i}\right)\right)$

are equal, where $A^{\prime \prime}=x A=x a P$. If these two values are equal, $\quad G W N \quad$ computes $T C_{i}=H\left(K_{G W N-U}\left\|I D_{i}\right\| T E_{i}\right) \quad, \quad P T C_{i}=T C_{i} \oplus$ $H\left(P W_{i}^{\text {new }}\left\|I D_{i}\right\| r_{i}\right)$, and updates its identity information table with the new entry $\left\{I D_{i}, T E_{i}\right\}$; otherwise, $G W N$ rejects the request. Finally, $G W N$ stores $\left\{H(\cdot), T E_{i}, P T C_{i}\right\}$ into a smart card and issues it to $U_{i}$.

- Step 3: After receiving the smart card, ${ }^{U_{i}}$ stores $r_{i}$ into it. Eventually, smart card has the following: $H(\cdot), T E_{i}, P T C_{i}$ and $r_{i}$.

\section{2) Registration phase for sensor nodes}

Assumpting that each node is preconfigured a password, and $G W N$ maintains the values $\left\{S T D_{j}, H\left(P W_{j}\right)\right\}$. The details of this phase are reviewed as follows.

- Step 1: ${ }^{S_{j}}$ randomly chooses a number $b \in Z_{p-1}^{*}$, computes $V I_{i}=H\left(T S_{2}\left\|H\left(P W_{i}\right)\right\| B \| B^{\prime}\right)$, where
$B=b P \quad, \quad B^{\prime}=b y=b x P \quad$. Then $S_{j}$ responds $\left\{S I D_{j}, T S_{2}, V I_{j}, B\right\}$ through a public channel.

- Step 2: When receiving the request message, $G W N$ firstly verifies the validity of $T S_{2}$. Then, $G W N$ retrieves $H\left(P W_{j}\right)$ according to $S I D_{j}$, computes $B^{\prime \prime}=x B=x b P$ and verifies whether $V I_{j}$ and $H\left(T S_{2}\left\|H\left(P W_{i}\right)\right\| B \| B "\right)$ are equal. If these two values are equal, $G W N$ further computes $T C_{j}=H\left(K_{G W N-S} \| S I D_{j}\right)$ $R E G_{j}=T C_{j} \oplus H\left(I S_{3}\left\|H\left(P W_{j}\right)\right\| B \mid B "\right) \quad$ and $V I_{G W N}=H\left(T C_{j} \| H\left(T S_{3}\left\|H\left(P W_{j}\right)\right\| B \| B "\right)\right)$ $G W N$ transimits $\left\{T S_{3}, R E G_{j}, V I_{G W N}\right\}$ to $S_{j}$.

- Step 3: Upon receiving the message, ${ }^{S_{j}}$ firstly verifies the validity of $T S_{3}$. Then, $S_{j}$ computes $T C_{j}=R E G_{j} \oplus \quad H\left(T S_{3}\left\|H\left(P W_{j}\right)\right\| B \| B^{\prime}\right) \quad$ and checks whether $V I_{G W N} \quad$ and $H\left(T C_{j} \| H\left(T S_{3}\left\|H\left(P W_{j}\right)\right\| B \| B^{\prime}\right)\right)$ are equal. If they are unequal, $S_{j}$ terminates the session; otherwise, it stores $T C_{j}$.

\section{B. Login and Authentication Phase}

- Step 1: $U_{i}$ inserts his smart card to a card reader, and inputs $I D_{i}$ and $P W_{i}$. The smart card calculates $T C_{i}=P T C_{i} \quad \oplus H\left(P W_{i}\left\|I D_{i}\right\| r_{i}\right)$. $U_{i}$ randomly chooses $c \in Z_{p-1}^{*}$ and a key $K_{i}$. Then $U_{i}$ calculates $C_{i}=c P \quad D_{i}=c y=c x P$

$D I D_{i}=I D_{i} \oplus H\left(C_{i} \| D_{i}\right)$

$P K S_{i}=K_{i} \oplus H\left(T C_{i}\left\|T S_{4}\right\| D_{i}\right)$

$E_{i}=H\left(H\left(I D_{i} \| T S_{4}\right) \oplus D_{i} \oplus P K S_{i} \oplus T C_{i}\right) \quad$, where $T S_{4}$ is the current timestamp. $U_{i}$ sends $\left\{D I D_{i}, C_{i}, P K S_{i}, T S_{4}, E_{i}\right\}$ to $G W N$.

- Step 2: When receiving this message, $G W N$ firstly verifies the validity of $T S_{4}$. Then, $G W N$ computes $D_{i}=x C=x c P \quad I D_{i}=D I D_{i} \oplus H\left(C_{i} \| D_{i}\right), T C_{i}=$ $H\left(K_{G W N-U}\left\|I D_{i}\right\| T E_{i}\right)$ and checks whether $E_{i}$ and $H\left(H\left(I D_{i} \| T S_{4}\right) \oplus D_{i} \oplus P K S_{i} \oplus T C_{i}\right)$ are equal. If they are unequal, $G W N$ rejects the request; otherwise, $\quad G W N \quad$ computes $K_{i}=P K S_{i} \oplus H\left(T C_{i}\left\|T S_{4}\right\| D_{i}\right)$. Then $G W N$ selects 
a sensor node $S_{j}$, and computes $T C_{j}=H\left(K_{G W N-s}\right.$

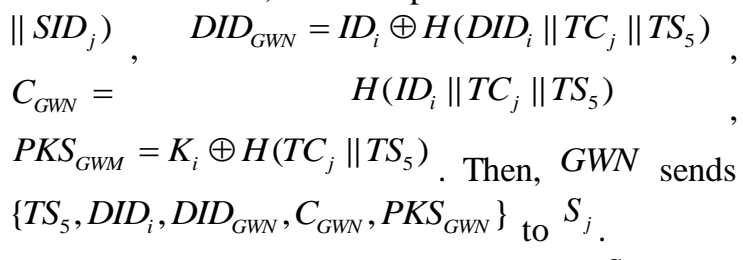

- Step 3: Upon receiving the message, ${ }^{S_{j}}$ firstly verifies the validity of $T S_{5}$. Then, $S_{j}$ computes $I D_{i}=D I D_{G W N} \oplus H\left(D I D_{i}\left\|T C_{j}\right\| T S_{5}\right)$, and checks whether $H\left(I D_{i}\left\|T C_{j}\right\| T S_{5}\right)$ and $C_{G W N}$ are equal. If they are equal, ${ }^{S_{j}}$ generates a random key $K_{j}$, computes $\quad K_{i}=P K S_{G W N} \oplus H\left(T C_{j} \| T S_{5}\right)$ $S K_{i j}=H\left(K_{i} \oplus K_{j}\right), C_{j}=H\left(K_{j}\left\|I D_{i}\right\| S I D_{j} \| T S_{6}\right)$, $P K S_{j} \quad=K_{j} \oplus H\left(K_{i} \| T S_{6}\right)$, and responds $\left\{S I D_{j}, T S_{6}, C_{j}, P K S_{j}\right\}$ to $U_{i}$; otherwise, ${ }^{S_{j}}$ rejects the request.

- Step 4: When receiving the validity of $T S_{6}, U_{i}$ computes $K_{j}=P K S_{j} \oplus H\left(K_{i} \| T S_{6}\right)$, and checks whether $C_{j}$ and $H\left(K_{j}\left\|I D_{i}\right\| S I D_{j} \| T S_{6}\right)$ are equal. If they are equal, $U_{i}$ confirms that $S_{j}$ and $G W N$ are authenticated, and further computes the shared session key $S K_{i j}=h\left(K_{i} \oplus K_{j}\right)$; otherwise, $U_{i}$ aborts this session.

\section{WEAKNESSES OF JIANG ET AL.'S SCHEME}

In this section, we will show that Jiang et al.'s scheme [10] is vulnerable to malicious user impersonation attack and node capture attack and suffer from forward security problem, low efficiency problem, no password change phase problem and time synchronization problem.

\section{A. Forward security problem}

Forward security problem means that once attacker obtains the master key stored by $G W N$ in some way, he/she will restore some previous session keys using the known master key and the information intercepted or eavesdropped from the public communicational channel. In this way, the attacker can easily decrypt the data transmitted in previous sessions.

In Jiang et al.'s scheme, suppose attacker obtains the master key $K_{G W N-S}$ and eavesdrops the mutual information $\left\{D I D_{i}, C_{i}, P K S_{i}, T S_{4}, E_{i}\right\} \quad\left\{T S_{5}, D I D_{i}, D I D_{G W N}, C_{G W N}\right.$, $\left.P K S_{G W N}\right\}$ and $\left\{S I D_{j}, T S_{6}, C_{j}, P K S_{j}\right\}$ in the authentication phase of some previous session, the attacker can restore the session key as follows:

- Computes $T C_{j}=H\left(K_{G W N-S} \| S I D_{j}\right)$, where $S I D_{j}$ have been obtained from $\left\{S I D_{j}, T S_{6}, C_{j}, P K S_{j}\right\}$.

- Computes $K_{i}=P K S_{G W N} \oplus H\left(T C_{j} \| T S_{5}\right)$, where $P K S_{G W N} \quad$ and $T S_{5}$ are from $\left\{D I D_{i}, C_{i}, P K S_{i}, T S_{4}, E_{i}\right\} \quad$ and $\left\{T S_{5}, D I D_{i}, D I D_{G W N}, C_{G W N}, P K S_{G W N}\right\}$ separately.

- Computes $K_{j}=P K S_{j} \oplus H\left(K_{i} \| T S_{6}\right)$, where $P K S_{j}$ and $T S_{6}$ are from $\left\{S I D_{j}, T S_{6}, C_{j}, P K S_{j}\right\}$.

- Restore the session key $S K_{i j}=H\left(K_{i} \oplus K_{j}\right)$.

\section{B. Malicious user impersonation attack}

Malicious user impersonation attack means that a malicious registered user can impersonate as other registered users to login the system, and access the sensed data under the name of other legitimate users. Suppose that a malicious user $A$ has captured the message $\left\{D I D_{i}, C_{i}, P K S_{i}, T S_{4}, E_{i}\right\}$ and collected $I D_{i}$ and $T E_{i}$ of a registered user $U_{i}$. Then $A$ can manipulate his smart card to launch the attack as follows.

- $A$ inserts his card and inputs $I D_{A}$ and $P W_{A}$. Then the card computes $T C_{A}=P T C_{A} \oplus H\left(P W_{A}\left\|I D_{A}\right\| r_{A}\right)$, and randomly chooses $c \in Z_{p-1}^{*}$ and a key $K_{A}$. Then $A$ calculates $C_{A}=c P \quad, \quad D_{A}=c y=c x P \quad, \quad D I D_{i}{ }^{\prime}=I D_{i} \oplus$ $H\left(C_{A} \| D_{A}\right), P K S_{i}{ }^{\prime}=K_{A} \oplus H\left(T C_{A}\left\|T S_{4}\right\| D_{A}\right)$, $E_{i}{ }^{\prime}=H\left(H\left(I D_{i} \| T S_{4}\right) \oplus D_{A} \oplus P K S_{A} \oplus T C_{A} . U_{i}\right.$ sends $\left\{D I D_{i}{ }^{\prime}, C_{A}, P K S_{i}{ }^{\prime}, T S_{4}, E_{i}{ }^{\prime}\right\}$ to $G W N$.

- When receiving the message, $G W N$ computes $D_{i}{ }^{\prime}=x C_{A}=x c P \quad I D_{i}=D I D_{i}^{\prime} \oplus H\left(C_{A} \| D_{i}^{\prime}\right)$ $T C_{i}{ }^{\prime}=H\left(K_{G W N-U}\left\|I D_{i}\right\| T E_{i}\right)$. It is easy to see that the value $H\left(H\left(I D_{i} \| T S_{4}\right) \oplus D_{i}{ }^{\prime} \oplus P K S_{i}{ }^{\prime} \oplus T C_{i}{ }^{\prime}\right)$ and $E_{i}{ }^{\prime}$ are equal. $G W N$ accepts $A$ as the legal user to access the data.Then, $G W N$ conducts the rest of operations in Step (2) of authentication and key agreement phase of Jiang et al.'s scheme and sends $\left\{T S_{5}, D I D_{i}, D I D_{G W N}, C_{G W N}, P K S_{G W N}\right\}$ to $S_{j}$.

- Upon receiving the message, $S_{j}$ follows the operations in Step (3) of authentication and key agreement phase of Jiang et al.'s scheme, and reponds $\left\{S I D_{j}, T S_{6}, C_{j}, P K S_{j}\right\}$ to $U_{i}$.

- $A$ intercepts the message, and computes $K_{j}=P K S_{j} \oplus H\left(K_{i} \| T S_{6}\right)$ and the shared session 
key $S K_{i j}=h\left(K_{i} \oplus K_{j}\right)$. Then $A$ can access the data of $S_{j}$.

\section{Sensor node capture attack}

Suppose a sensor node $S_{j}$ was captured by an attacker $A$. Since the capabilities of computation and storage of the sensor node $S_{j}$ are both limited, $A$ can easily extract the data stored in the sensor node $S_{j}$, especially the value $T C_{j}$. Once the attacker $A$ obtained $T C_{j}$ and eavesdrops the mutual information $\left\{T S_{5}, D I D_{i}, D I D_{G W N}, C_{G W N}, P K S_{G W N}\right\}$ and $\left\{S I D_{j}, T S_{6}, C_{j}, P K S_{j}\right\}$ in the authentication phase of some previous session, the attacker $A$ can restore the session key as follows:

- $A_{\text {computes }} I D_{i}=D I D_{G W N} \oplus H\left(D I D_{i}\left\|T C_{j}\right\| T S_{5}\right)$, where $D I D_{G W N}, D I D_{i}$ and $T S_{5}$ have been obtained from the message $\left\{T S_{5}, D I D_{i}, D I D_{G W N}, C_{G W N}, P K S_{G W N}\right\}$

- $A$ computes $K_{i}=P K S_{G W N} \oplus H\left(T C_{j} \| T S_{5}\right)$, where $P K S_{G W N}$ and $T S_{5}$ are from $\left\{T S_{5}, D I D_{i}, D I D_{G W N}\right.$, $\left.C_{G W N}, P K S_{G W N}\right\}$.

- $A$ computes $K_{j}=P K S_{j} \oplus H\left(K_{i} \| T S_{6}\right)$, where $P K S_{j}$ and $T S_{6}$ are from $\left\{S I D_{j}, T S_{6}, C_{j}, P K S_{j}\right\}$.

- $A$ computes the session key $S K_{i j}=H\left(K_{i} \oplus K_{j}\right)$.

\section{Low efficiency in wrong password detection}

If the legal user $U_{i}$ inputs a wrong password by mistake, this wrong password will not be detected until the remote gateway node $G W N$ verifies whether $E_{i}$ and $H\left(H\left(I D_{i} \| T S_{4}\right) \oplus D_{i} \oplus P K S_{i} \oplus T C_{i}\right)$ are equal in step 2 of the login and authentication phase. Therefore, Jiang et al.'s scheme is low efficient to detect the user's wrong password.

\section{E. No password change phase}

In Jiang et al.'s scheme, there is no password change phase. Actually, it is not difficult to add this phase. When the user $U_{i}$ wants to change his/her password, he/she inserts the smart card into a card reader, inputs the identity $I D_{i}$ and password $P W_{i}$, then calls for changing password. ${ }^{U_{i}}$ will select and input a new password $P W_{\text {new }}$. And then the smart card computes $\quad P T C_{\text {new }}=P T C_{i} \oplus H\left(P W_{i}\left\|I D_{i}\right\| r_{i}\right) \oplus$ $H\left(P W_{\text {new }}\left\|I D_{i}\right\| r_{i}\right)$, and replaces $P T C_{i}$ with $P T C_{\text {new }}$. As such, the password is changed.

However, since no wrong password detection mechanism is designed in the smart card, the password change phase would suffer from the following weakness. If an attacker $A$ stole user $U_{i}$ 's smart card for a short time, he/she inserts $U_{i}$ 's smart card into a card reader, enters the $I D_{i}$ and an arbitrary password $P W_{a}$, and calls for changing password. Then $A$ enters an arbitrary new password $P W_{a}^{*}$. The smart card will compute $P T C_{a}^{*}=P T C_{i} \oplus H\left(P W_{a} \| I D_{i}\right.$ $\left.\| r_{i}\right) \oplus H\left(P W_{a}^{*}\left\|I D_{i}\right\| r_{i}\right)$, which yields $P T C_{a}^{*}=P T C_{i} \oplus$ $H\left(P W_{a}\left\|I D_{i}\right\| r_{i}\right) \oplus H\left(P W_{a}^{*}\left\|I D_{i}\right\| r_{i}\right)=T C_{i} \oplus H\left(P W_{i} \| I D_{i}\right.$ $\left.\| r_{i}\right) \oplus H\left(P W_{a}\left\|I D_{i}\right\| r_{i}\right) \oplus H\left(P W_{a}^{*}\left\|I D_{i}\right\| r_{i}\right)$, and then replaces $P T C_{i}$ with $P T C_{a}^{*}$ without any checking. Later, the legal user $U_{i}$ 's succeeding login requests will be denied.

\section{F. Time synchronization problem}

Since time stamp was adopted in the login and authentication phase of Jiang et al.'s scheme, it is inevitable that there is a probability of time synchronization problem between user and gateway node. Also same problem can be occurred between gateway node and sensor nodes during communication.

\section{CONCLUSIONS}

In this paper, we analyze an untraceable temporalcredential-based two-factor authentication scheme using ECC for WSNs proposed by Jiang et al. in 2016, and point out the scheme can not resist malicious user impersonation attack, sensor node capture attack and suffer from forward security problem, low efficiency problem, no password change phase problem and time synchronization problem.

\section{ACKNOWLEDGMENT}

This work was partially supported by the Doctoral Fund of University of Jinan (Granted No. XBS1455), the project of Shandong Natural Science Foundation (Granted No. ZR2013FM009), and the Youth Fund of Shandong Women's University (Granted No. 2014ZDX15).

\section{REFERENCES}

[1] M. L. Das. Two-factor user authentication in wireless sensor networks. IEEE Trans. Wireless Communication, 2009, 8(3): 10861090

[2] M. K. Khan, K. Alghathbar. Cryptanalysis and security improvement of two-factor user authentication in wireless sensor networks. Sensors, 2010: 2450-2459.

[3] D. J. He, Y. Gao, S. Chan, et al.. An enhanced two-factor user authentication scheme in wireless sensor networks. Ad Hoc Sensor Wireless Netw., 2010, 10(4): 1-11.

[4] T. H. Chen, W. K. Shih. A robust mutual authentication protocol for wireless sensor networks. ETRI J., 2010, 32(5): 704-712

[5] C. C. Chang, H. D. Le. A provably secure, efficient, and flexible authentication scheme for ad hoc wireless sensor networks. IEEE Trans. Wireless Communication, 2016, 15(1): 357-365.

[6] R. Amin, G. P. Biswas. A secure light weight scheme for user authentication and key agreement in multi-gateway based wireless sensor networks. Ad Hoc Networks, 2016, 36(1): 58-80. 
[7] P. Kumar, A. Gurtov, M. Ylianttila, et al.. A strong authentication scheme with user privacy for wireless sensor networks. ETRI Journal, 2013, 35(5): 889-899.

[8] K. Xue, C. Ma, P. Hong, R. Ding. A temporal-credential-based mutual authentication and key agreement scheme for wirless sensor networks. Journal of Network and Computer Applications, 2013, 36(1): 316-323.
[9] D. He, N. Kumar, N. Chilamkurti. A secure temporal-credentialbased mutual authentication and key agreement scheme with pseudo identity for wirless sensor networks. Inf. Sci., 2015, 321: 236-277.

[10] Q. Jiang, J. Ma, F. Wei, et al.. An untraceable temporal-credentialbased two-factor authentication scheme using ECC for wireless sensor networks. Journal of Network and Computer Applications, http: // dx.doi.org/10.1016/j.jnca.2016.10.001. 